\title{
Développer la littératie des apprenants grâce à l'intégration des TIC
}

\section{CHRONIQUE • Milieu scolaire}

L'intégration des technologies de l'information (TIC) en éducation peut maintenant permettre à l'enseignant et aux différents professionnels qui interviennent auprès des élèves d'avoir accès aux mêmes commentaires métalinguistiques (Gombert, 1990) de ceux-ci après l'enseignement systématique de notions, de concepts ou de stratégies liés au développement de la littératie.

Développer la littératie de l'ensemble des apprenants constitue un incontournable du système scolaire au XXI ${ }^{\mathrm{e}}$ siècle. « La littératie est l'aptitude à comprendre et à utiliser l'information écrite dans les différents genres de textes proposés en vue d'accroitre les compétences pour pouvoir acquérir de nouvelles connaissances, résoudre des problèmes et former son sens critique » (Granger et Dubé, 2014). Pour développer la littératie, avoir accès aux connaissances déclaratives, conditionnelles et procédurales de tous les élèves, et plus particulièrement des élèves en difficulté d'apprentissage, devient un atout majeur pour les enseignants et pour les professionnels.

Une exploration menée en 2015 auprès d'apprenants de 12 et 13 ans nous a permis de constater tout le potentiel de ce travail partagé grâce à certains outils numériques en ligne. En effet, dans le cadre d'une recherche menée dans notre milieu de travail, nous avons utilisé une application de partage nommée Explain everything qui a permis tant à l'enseignant, au conseiller pédagogique et au chercheur d'avoir accès en même temps aux différentes traces d'apprentissage de l'élève. Cette façon de 
procéder a permis à l'équipe d'avoir accès aux commentaires métagraphiques de chacun des apprenants concernés par l'essai, soit 44 élèves de la première secondaire.

Des applications telles qu'Explain everything permettent maintenant de consigner en ligne les enregistrements audio et vidéo des élèves afin d'analyser ces mêmes données, analyse commune qui améliorera la cohérence dans les interventions à différents niveaux. Par exemple, dans une école, cette façon de procéder devrait permettre à l'enseignant, à l'enseignant-ressource et à l'orthopédagogue d'intervenir plus efficacement et plus rapidement, en offrant une rétroaction ciblée en fonction des conceptions erronées des apprenants en difficulté (Whitten, Esteves et Woodrow, 2012).

Quand plusieurs intervenants travaillent auprès du même apprenant sur le plan des apprentissages, le partage des traces recueillies en classe permet à ceux-ci de connaitre immédiatement ce qui sera à travailler lors de la prochaine rencontre avec l'élève concerné. Ce partage permet donc que tous les intervenants travaillent à partir de la même trace plutôt que de dédoubler celle-ci. Cela permet aussi aux intervenants de gagner du temps de concertation puisque tous ont accès à la même information en simultané. Il en résulte donc un gain de temps, temps qui sera réinvesti auprès de l'apprenant.

Permettre à ces intervenants d'avoir accès aux commentaires métalinguistiques des élèves après l'enseignement systématique de notions, de concepts ou de stratégies offre la possibilité à ceux-ci d'intervenir plus efficacement en offrant une rétroaction ciblée en fonction des conceptions erronées des apprenants. Nous savons qu'une rétroaction efficace constitue un facteur contribuant à la réussite des apprentissages de l'élève (Hattie, 2009).

Il y a quelques années seulement, cette façon de procéder apparaissait inimaginable à cause du temps exigé pour ce faire. Maintenant, grâce à l'intégration des TIC en éducation, cela devient possible puisque les outils de consignation en ligne permettent de recueillir les enregistrements audio et vidéo des apprenants et d'analyser ces données afin d'intervenir rapidement si certaines conceptions s'avèrent erronées.

\section{Comment procède-t-on?}

En classe, tous les apprenants doivent réinvestir leur connaissance de ce qui a été enseigné par rapport à une tâche proposée par l'enseignant, tâche réalisée à l'aide d'une application comme Explain everything. Cette dernière permet à l'utilisateur d'annoter, d'animer et de narrer des explications et des présentations. Celles-ci peuvent donc ensuite être partagées à un auditoire précis.

Dans le cadre de ce projet, l'auditoire est constitué des différents intervenants. Ce partage, sous forme de vidéo, permet donc aux intervenants de suivre en collaboration l'évolution de la compréhension des élèves ciblées. La régulation de toutes les interventions sera donc davantage concertée.

Cette nouvelle façon de faire change les pratiques des différents intervenants puisque toutes les interventions sont traitées plus étroitement avec la planification des apprentissages de l'enseignant en classe. 


\section{Quels sont les principaux gains?}

Le partage en ligne des commentaires métalinguistiques des apprenants entre les intervenants concernés permet de suivre leur progression en temps réel et évite le dédoublement des traces demandées à l'élève. Ces traces peuvent aussi être accessibles aux parents, leur permettant ainsi de contribuer à l'apprentissage de leur enfant. Un gain de temps, tant pour l'élève que pour les différents intervenants, devient également possible grâce à ce moyen d'intervention. En effet, nous mettons trop souvent de côté le temps devant être alloué à la concertation, faute de disponibilités dans les différents horaires des intervenants (Ministère de l'Éducation, 2003). Aussi, dans un contexte où l'orthopédagogue ne voit l'élève qu'une fois toutes les deux semaines, cette façon de faire permet à celui-ci de cibler encore plus efficacement ses interventions.

L'intégration des TIC en éducation est un vecteur de changement dans ce domaine. Les différents outils (principalement les outils mobiles) nous permettent de travailler autrement afin que les interventions des différents intervenants soient plus cohérentes aux yeux de l'apprenant, lui permettant ainsi d'augmenter son niveau de littératie plus rapidement.

\section{Références}

Gombert, J.-É. (1990). Le développement métalinguistique. Paris : PUF.

Granger, N. et Dubé, F. (2014). Améliorer le rapport à l'écrit d'élèves en difficulté au secondaire : des choix pédagogiques qui font toute la différence. Repéré à http://www.aqpf.qc.ca/contentDocuments/0BC6372B-7054-D219-67E4E83C82860C36.ppt

Hattie, J. (2009). Classement de Hattie : liste de facteurs pour la réussite scolaire. Repéré à http://visible-learning.org/fr/john-hattie-classement-facteurs-reussite-apprentissage/

Ministère de l'Éducation. (2003). Les difficultés d'apprentissage à l'école : Cadre de référence pour guider l'intervention. Repéré à http://www.education.gouv.qc.ca/fileadmin/site web/documents/dpse/adaptation serv comp1/19-7051.pdf

Whitten, É., Esteves, K. J. et Woodrow, A. (2012). La réponse à l’intervention : un modèle efficace de différenciation. Montréal, QC : Chenelière Éducation.

\section{Pour citer cet article}

Fradette, M. (2016). Développer la littératie des apprenants grâce à l'intégration des TIC. Formation et profession 24(2), 7577. http://dx.doi.org/10.18162/fp.2016.a97 